\title{
High throughput in silico identification of novel phytochemical inhibitors for the master regulator of inflammation (TNF $\alpha$ )
}

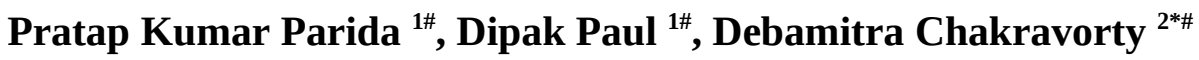 \\ ${ }^{1}$ Noor Enzymes Private Limited, 37-B, Darga Road, Kolkata - 700017, India \\ ${ }^{2}$ Novel Techsciences (OPC) Private Limited, 37-B, Darga Road, 1st Floor, Kolkata - 700017, \\ India \\ * Corresponding author: \\ Debamitra Chakravorty, PhD (Project Lead - Computational Biology) \\ Novel Techsciences (OPC) Private Limited, 37-B, Darga Road, 1st Floor, Kolkata - 700017, \\ India \\ E-mail: dc@noveltechsciences.com \\ \# All the authors have contributed equally to the paper.
}

\begin{abstract}
The over expression of Tumor necrosis factor- $\alpha$ (TNF $\alpha)$ has been implicated in a variety of disease and is classified as a therapeutic target for inflammatory diseases (Crohn disease, psoriasis, psoriatic arthritis, rheumatoid arthritis). Commercially available therapeutics are biologics which are associated with several risks and limitations. Small molecule inhibitors and natural compounds (saponins) were identified by researchers as lead molecules against TNF $\alpha$, however, they were often associated with high IC50 values which can lead to their failure in clinical trials. This warrants research related to identification of better small molecule inhibitors by screening of large compound libraries. Recent developments have demonstrated power of natural compounds as safe therapeutics, hence, in this work, we have identified TNF $\alpha$ phytochemical inhibitors using high throughput in silico screening approaches of 6000 phytochemicals followed by 200 ns molecular dynamics simulations and relative binding free energy calculations. The work yielded potent hits that bind to TNF $\alpha$ at its dimer interface. The mechanism targeted was inhibition of oligomerization of TNF $\alpha$ upon phytochemical binding to restrict its interaction with TNF-R1 receptor. MD simulation analysis (Principal component analysis, Dynamic Cross Correlation Matrices, Free energy landscapes) resulted in identification of two phytochemicals that showed stable protein-ligand conformations over time. The two compounds were triterpenoids: Momordicilin and Nimbolin A with relative binding energy- calculated by MM/PBSA to be $-190.5 \mathrm{~kJ} / \mathrm{Mol}$ and $-188.03 \mathrm{~kJ} / \mathrm{Mol}$ respectively. Therefore, through this work it is being suggested that these phytochemicals can be used for further in vitro analysis to confirm their inhibitory action against TNF $\alpha$ or can be used as scaffolds to arrive at better drug candidates.
\end{abstract}

Keywords: Virtual screening, Docking, MD simulations, TNF- $\alpha$, Anti-inflammatory, Phytochemicals. 


\section{Introduction}

Tumor necrosis factor $\alpha(\mathrm{TNF} \alpha)$, also known as cachectin, is a well-established therapeutic target for various inflammatory and autoimmune related diseases [1]. TNF $\alpha$ is a $25 \mathrm{kDa}$ transmembrane protein and $17 \mathrm{kDa}$ when secreted. The autoimmune diseases related to unregulated $\mathrm{TNF} \alpha$ activity include inflammatory bowel disease, diabetes, rheumatoid arthritis, systemic sclerosis, systemic lupus erythematosus, multiple sclerosis, diabetes, asthma, ankylosing spondylitis, cancer and AIDS [2,3]. The pleiotropic cytokine TNF $\alpha$ is produced by macrophages and by several other proinflammatory cells, including monocytes, dendritic cells, B cells, CD4 ${ }^{+}$cells, neutrophils, mast cells and eosinophils, and the structural cells [4]. The signalling pathway TNF $\alpha$ involves activation of the nuclear factor $\kappa \mathrm{B}$, which interacts with the DNA to increase transcription of IL1B, IL6, IL8, and TNF $\alpha$ itself [3]. It is thus clear that inhibition of TNF $\alpha$ activity will benefit TNF $\alpha$ mediated diseases.

The binding of TNF $\alpha$ trimer to its receptors effects the activation of transcription factors related to cell proliferation, differentiation, cell survival or apoptosis [5]. A series of synthetic antibodies (infliximab, etanercept, adalimumab, certolizumab and glolimumab) have been developed for the treatment of cancer and autoimmunity [6,7]. These biologics are often associated with high cost, poor clinical response, and requirement for intravenous administration. Small molecule inhibitors are relatively cheaper and can be taken orally. Small molecule therapies for TNF $\alpha$ have not led to approved products [8]. Therefore, the identification of small molecules that can inhibit $\mathrm{TNF} \alpha$-regulated pathways is a promising research area that has lately received much attention. However, to this point, there have been no publications of small-molecule inhibitors past the proof-of-concept stage of targeting TNF $\alpha$ [9]. Disruption of TNF $\alpha$ binding to TNFR1, has been the goal in the development of therapeutics $[10,11]$.

Majority of the small molecules target TNF by down regulating its expression. TNF $\alpha$ binds to its receptor in a trimeric form, its potential inhibitors can be selected for preventing the formation of trimer by stabilizing the inactive dimeric form [12]. A limited number of compounds is reported to directly disrupt this interaction [13]. Suramin and its analogues were reported to inhibit $\mathrm{TNF} \alpha$ interaction with its receptor by causing inhibition of oligomerization of the TNF $\alpha$ trimer with an IC50 of $0.65 \mathrm{mM}[14,15]$. Compounds similar in 
structure with suramin: Trypan blue and Evans blue were reported with IC50 of 0.75 and 1 $\mathrm{mM}$, respectively. All the three compounds were reported to make contact of Arg103, Tyr119 and Lys98 in TNF $\alpha$ structure [16]. These residues have been reported to be important for trimer association [17]. Further development of small molecule inhibitor was by He et al. [18] who synthesized SPD305 which inhibits TNF $\alpha$ interaction with its receptor with an IC50 of $22 \mu \mathrm{M}$ measured by ELISA. SPD305 also led to deoligomerization of TNF $\alpha$ making 16 contacts with the interface residues including Tyr59 and Tyr119. There residues were reported to be important for trimer contact [18]. However, this molecule is still a proof of concept and has not been perused further for in vivo testing and is related with high toxicity [19]. This may be related to its high IC50 value by ELISA [9]. Progressive research led Buller et al. [20] to identify compounds that inhibited TNF $\alpha$ by using DNA-encoded library. They identified analogues with dichloro-benzophenone moiety to inhibit TNF $\alpha$ at concentrations higher than $300 \mu \mathrm{M}$ [20]. Natural compound inhibitors for TNF $\alpha$ were reported by Shah et al. [21] from the methanolic extract of Parthenium hysterophorus. The compounds were saponins (terpenoid class of natural compounds). These class of compounds have been reported to have including anti-inflammatory activity [22]. It was proposed that these compounds led to inhibition of TNF $\alpha$ by deoligomerization like the compound SPD305 [21]. Choi and co-authors [12] virtually screened a library of 240,000 compounds. The three top compounds all shared a pyrimidine-2,4,6-trione moiety and involved all involved hydrophobic interactions with Tyr59, Tyr119 and Tyr159. Further research led to the identification of hydrophobic natural products that could mimic the binding of SPD305 [23]. Quinuclidine with an IC50 50 $\mu \mathrm{M}$ and indoloquinolizidine with an IC50 $10 \mu \mathrm{M}$ were reported as TNF inhibitors. Their binding was like SPD305 [23]. Wua et al. [24] reported that curcumin can bind to TNF $\alpha$ by molecular docking studies. Melagraki et al. [13] identified two molecules T23 and T8 to be direct inhibitors of TNF $\alpha$.

It is to be noted here that the potency of TNF for TNF receptor 1 (TNFR1) is picomolar range [8].The reported small molecules showed potential inhibitory activity with TNF $\alpha$ but they have high IC50 values in vitro. This may lead to their failure in clinical trials. This warrants research related to identification of better small molecule inhibitors. Recent developments have demonstrated power of natural compounds as drugs [25-29]. Inspired by this, in this work the disassembly of TNF $\alpha$ similar to that proposed by He et al. [18] by phytochemicals were analysed by performing virtual screening of 6000 natural compounds from medicinal plants reported to have anti-inflammatory activities. This was followed by 
calculation of relative binding free energies of the top hits to identify the best phytochemical inhibitors.

\section{Materials and methods}

\subsection{Virtual screening of phytochemicals from medicinal plants}

The crystal structure of TNF $\alpha$ (2az5) with resolution $2.1 \AA$ was downloaded from RCSB PDB. The structure is with a small molecule inhibitor (SPD-305) which was reported to inhibit TNF $\alpha$ activity in biochemical and cell-based assays with median inhibitory concentrations of 22 and 4.6 micromolar, respectively [18]. The ligand binding site of TNF $\alpha$ was predicted using the PDBsum server [30]. The ligand binding site lies between chain A (Val91, Asn92, Leu93, Phe124) and chain B (His15, Val17, Ala18, Pro20, Arg32 Ala33, Asn34, Ala35, Tyr119, Phe144, Glu146, Ser147, Gly148, Gln149 and Val150). MGLTools1.5.6 was used for the generation of grid file and the docking file parameters. Virtual screening of 6000 phytochemicals from medicinal plants, reported to be effective against inflammatory diseases, was performed using the Raccoon plug-in of Autodock 4.2.6. The configurations files generated from MGLTools-1.5.6 are incorporated in the Raccoon plug-in package. The virtual screening was run with 10 Lamarkian Genetic Algorithm (LGA) runs with the default parameters of Autodock 4.2.6. Phytochemicals with lowest binding energies (kcal/mol) were identified and subjected for re-docking for 100 LGA runs. The inhibitor obtained from the crystal structure (SPD305) was also docked as a control for 100 LGA runs. The docked complexes were visualized using BIOVIA Discovery Studio Visualiser [31].

\subsection{Toxicity analysis of top scoring phytochemicals by in silico approaches}

Adsorption, Distribution, Metabolism, Excretion (ADME) properties were calculated using SwissADME [32-34]. Toxicity profiles (T) were annotated using the vNN web server [35]. ADME/T associated properties of Gastro Intestinal absorption (GI), Blood Brain Barrier permeation (BBB), CYP inhibition, pharmacokinetic properties like Lipinski rule of 5 [36], PAINS [37] and Brenk [38] and Lead likeness were calculated [39].

\subsection{Molecular dynamics simulations and trajectory analysis}

The top scoring phytochemicals obtained by virtual screening and the control inhibitor were analysed by all atom molecular dynamics (MD) simulations for $1 \mu$ s (Table 1 ). All the simulations were carried out with Gromacs 2020.2 software package with all atom 
AMBER99SB-ILDN force field [40]. The force field parameters for the phytochemicals and the repurposed drugs were generated by ACPYPE (AnteChamber PYthon Parser interface) [41]. Complex charges were neutralized with sodium and chloride ions. Simulation was conducted at $300 \mathrm{~K}$ under a pressure of 1 bar. Each system was minimized with 5,000 steps by steepest descent algorithm. Particle-Mesh-Ewald summation (PME) was used to calculate electrostatic interactions [42]. In NVT and NPT ensembles the systems were equilibrated for $1 \mathrm{~ns}$ using position restraint simulations of $1000 \mathrm{~kJ} \mathrm{~mol}^{-1} \mathrm{~nm}^{-2}$. This was followed by a no restraint production run for 200 ns. Post-MD analyses included root mean square deviation (RMSD), root mean square fluctuations (RMSF), the radius of gyration (Rg) and hydrogen bond occupancy. PCA on C $\alpha$ atomic coordinates was performed using R Studio and Bio3d [43]. The eigenvectors and eigenvalues and their projection along with the first two principal components were analysed [44]. It was assumed that a stable drug bound complex will undergo fewer dynamic fluctuations due to enhancement of rigidity of the drug bound site. Further, DCCM C $\alpha$ atomic coordinates was performed using the Bio3D package. Molecular Mechanics - Poisson Boltzmann Surface Area (MM-PBSA) was applied on snapshots obtained from MD trajectory to estimate the relative binding free energy $\Delta G_{\mathrm{b}}$ using the GROMACS tool g_mmpbsa [45,46]. The Free Energy landscape was obtained from PC1 and PC2 projections using g_sham tool in GROMACS MD package. The global minimum conformations were extracted from the FEL bins. These structures acted as representatives for network centrality measures and protein interaction network analysis for cliques. High centrality measures correlate to a node's capacity to effect protein function. According to graph theory, the more connected a node (amino acid residue) to others the higher its degree centrality. More details about the network centrality measures can be found in the work of Chakrabarty et al. 2016. A high degree node has high betweenness centrality because many of the shortest paths may pass through that node. These properties were expected for the phytochemical inhibitor- TNF $\alpha$ complexes to be like the control-TNF $\alpha$ complex.

\section{Results}

\subsection{Virtual screening of phytochemicals for TNF $\alpha$}

KEGG pathway analysis shows that TNF $\alpha$ is binds to two different receptors, TNFR1 and TNFR2, and activate caspase-mediated apoptosis, NF-kB, activator protein-1, MAPK and ERK signalling and p13k-Akt signalling (Figure 1). Crystal structures TNF $\alpha$ have a high degree of anti-parallel $\beta$-sheet [9]. The core consists of $4 \beta$-strands and the quaternary 
structure is a homo-trimer [9]. The inter-chain contact is formed by hydrophobic residues (Tyr119, Leu57 and Leu157). Hydrophobic residue interactions also exist between Tyr59, Tyr119 and Gly153 of one subunit and Phe124 of a neighbouring subunit. A salt bridge also exists between Lys11 of one subunit and Leu156 of another subunit [47]. Therefore, these residues were considered as the binding site of TNFa. Docking grid was generated considering residues to be present in the binding pocket.

Figure 1: TNF $\alpha$ in inflammation related diseases. The signalling pathway was adapted from KEGG. The diseases associated have been illustrated in blue circles. The biologics available for TNF are illustrated in orange circle. The cartoon representation of the structure of TNF $\alpha$ in complex with a small molecule inhibitor (represented in cyan ball and stick) was obtained using PyMol molecular graphics tool. The bar graph depicts the \% of compounds from the medicinal plants that docked to TNF $\alpha$ with docking score lesser than the bound control ligand obtained from RCSB PDB (PDB ID: 2az5).

A total of 6000 compounds were screened against TNF $\alpha$. A total of 742 compounds showed lower docking score as compared to the control compound $(-8.64 \mathrm{~kJ} / \mathrm{Mol})$. The medicinal plants to which these 742 compounds belong were quantified according to the percentage of hits obtained for their compounds (Figure 1A). It was interesting to observe that majority of the phytochemicals that were able to dock to TNF $\alpha$ binding site (8.35\%) were from Momordica charantia. The other important medicinal plants whose $>2 \%$ compounds docked to TNF were observed to be from Azadirachta indica, Withania somnifera, Mangifera indica, Euphorbia hirta and Nigella sativa. The top 10 scoring compounds with docking score $<-10 \mathrm{~kJ} / \mathrm{Mol}$ were from Momordica charantia, Azadirachta indica, Strychnos nux-vomica, Bauhinia purpurea, Swertia chirayita, Solanum torvum and Mangifera indica (Table 1).

Table 1: Top 10 phytochemicals docked to TNF $\alpha$

\begin{tabular}{|l|l|l|l|l|l|}
\hline Sl. No. & Compound Name & $\begin{array}{l}\text { Score (kJ/ } \\
\text { Mol) }\end{array}$ & Source & 2D structure & $\begin{array}{l}\text { MRTD } \\
\text { dose } \\
\text { mg/day }\end{array}$ \\
\hline
\end{tabular}




\begin{tabular}{|c|c|c|c|c|}
\hline 1 & Goyaglycoside-a & -11.7 & $\begin{array}{l}\text { Momordica } \\
\text { charantia }\end{array}$ & 99 \\
\hline 2 & Charantoside I & -11.55 & $\begin{array}{l}\text { Momordica } \\
\text { charantia }\end{array}$ & 94 \\
\hline 3 & Nimbolin A & -11.12 & $\begin{array}{l}\text { Azadirachta } \\
\text { indica }\end{array}$ & 195 \\
\hline 4 & Momordicilin & -11.06 & $\begin{array}{l}\text { Momordica } \\
\text { charantia }\end{array}$ & 109 \\
\hline 5 & Swertanone & -10.79 & $\begin{array}{l}\text { Swertia } \\
\text { chirayita }\end{array}$ & 183 \\
\hline 6 & Solanolactoside A & -10.66 & $\begin{array}{l}\text { Solanum } \\
\text { torvum }\end{array}$ & 79 \\
\hline
\end{tabular}




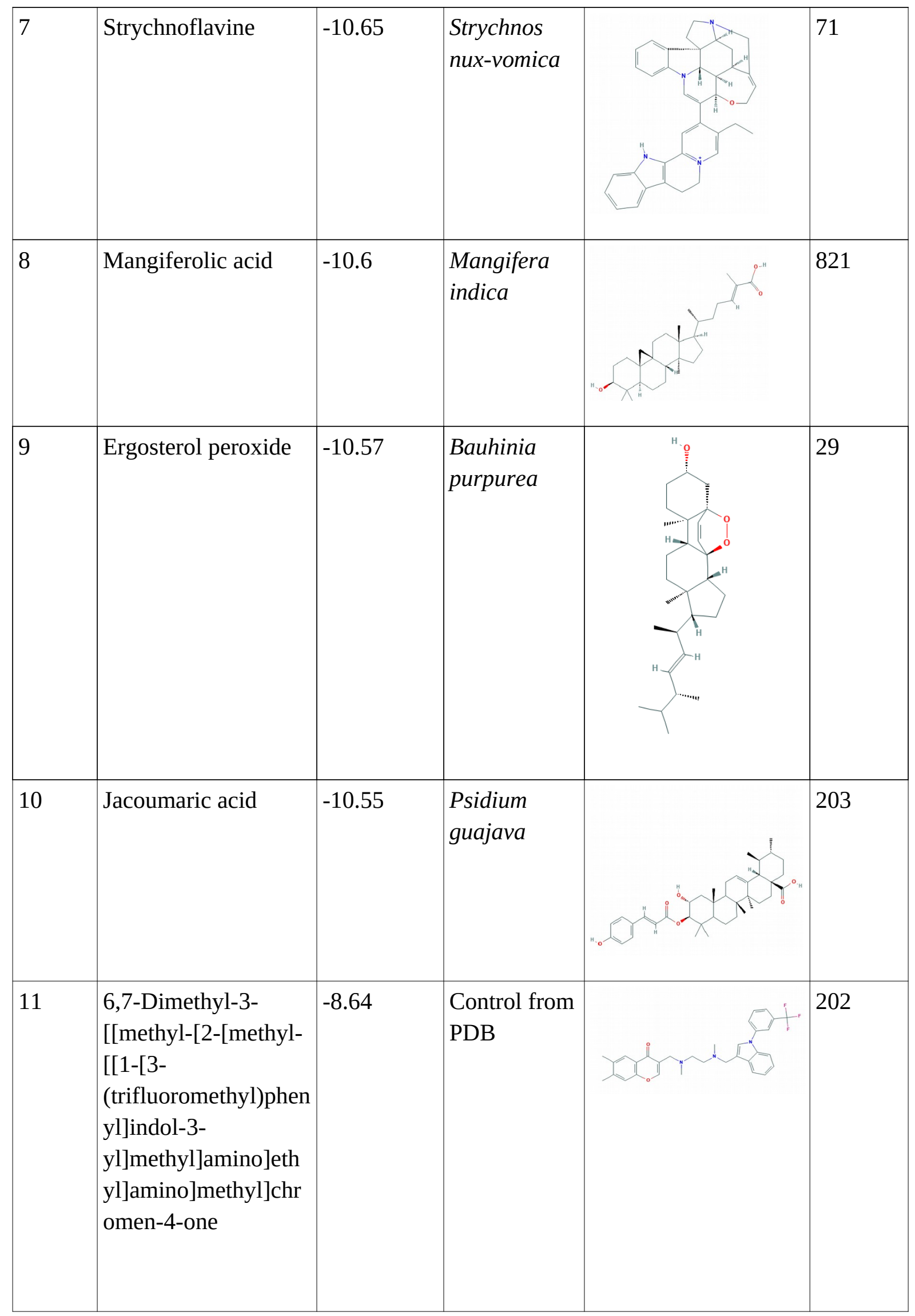


The docked complexes and the residues in TNF $\alpha$ interacting with the top four compounds and the control drug have been illustrated in Figure 2. The toxicity profiles calculated by vNN-ADMET have been presented in Appendix I. The top three compounds with good docking scores and following standard toxicity profile along with the control were submitted for MD simulation analysis production run of 200 ns. The significant pharmacokinetic parameters for ADME/T associated properties of Gastrointestinal absorption (GI), Blood Brain Barrier permeation (BBB), CYP inhibition, pharmacokinetic properties like Lipinski rule of 5, PAINS, Brenk and Lead likeness showed that these compounds were not toxic (Supplementary Materials).

Figure 2: Docked complexes and residue interaction 2D image of phytochemicals and control drug with TNF $\alpha$ having lead likeness 3. A) Control, B) Goyaglycoside-a, C) Charantoside I, D) Nimbolin A, E) Momordicilin.

Analysis of Figure 2 has been presented in Table 2. The phytochemicals formed more hydrogen bonded interactions with residues of chain A and chain B in the TNF $\alpha$ structure in comparison to the control. $\pi$-alkyl and $\pi-\pi$ stacking interactions were also observed to dominate the binding of the phytochemicals and the control drug to TNF $\alpha$. The residues interacting with the phytochemicals and the control drug were Tyr119 B, Lys98 B, Pro117 B, Lys98 A, Ser60 A, Leu120 A, Tyr151 A, Tyr59 A, Leu57 A, Tyr 119 A, Tyr151 B, Gly121 A, Tyr59 B, Leu57 B, Gly121 B, Ile58 A, Leu 120 A, Tyr119 B, Tyr119 A and Leu120 B.

Table 2: Molecular interactions obtained after docking of phytochemicals and control drug with the homodimer of TNF $\alpha$

\begin{tabular}{|c|c|c|c|}
\hline H-bonds & 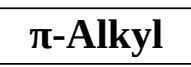 & $\pi-\pi$ & Halogen \\
\hline \multicolumn{4}{|c|}{ Goyaglycoside-a } \\
\hline Tyr119 B & Tyr151 A & - & - \\
\hline Lys98 B & Tyr59 A & - & - \\
\hline Pro117 B & Leu57 A & - & - \\
\hline Lys98 A & Tyr119 A & - & - \\
\hline Ser60 A & - & - & - \\
\hline Leu120 A & - & - & - \\
\hline \multicolumn{4}{|c|}{ Charantoside I } \\
\hline Tyr119 B & Tyr151 A & - & - \\
\hline Pro117 B & Tyr59 A & - & - \\
\hline Lys98 A & Leu57 A & - & - \\
\hline Lys98 B & Tyr119 A & - & - \\
\hline \multicolumn{4}{|c|}{ Nimbolin A } \\
\hline
\end{tabular}




\begin{tabular}{cccc}
\hline Tyr151 B & Tyr59 B & Leu57 A & - \\
Gly121 A & Leu57 B & Tyr59 A & - \\
\hline \multicolumn{5}{c}{ Momordicilin } \\
\hline Tyr151 B & Tyr59 B & - & - \\
- & Tyr119 A & - & - \\
- & Tyr119 B & - & - \\
- & Leu57 A & - & - \\
\hline \multicolumn{5}{c}{ Control } \\
\hline Gly121 B & Tyr119 B & Tyr119 A & Tyr119B \\
Ile 58 A & - & Tyr119 B & - \\
Leu 120 A & - & Leu120 B & - \\
- & - & Tyr59 B & - \\
\hline
\end{tabular}

- Not found

\subsection{Molecular dynamics simulation trajectory analysis}

The 200 ns trajectory analysis of the top four docked complexes and the control. Figure 3A illustrates stable RMSD over $200 \mathrm{~ns}$ for all the trajectories (average: $0.30 \mathrm{~nm}$ ). The average RMSD values for the phytochemicals were Goyaglycoside-a: $0.26 \mathrm{~nm}$, Nimbolin A: $0.24 \mathrm{~nm}$, Charantoside I: $0.28 \mathrm{~nm}$, Momordicilin: $0.29 \mathrm{~nm}$ and the control: 0.27 . This shows a stable binding profile of the phytochemicals and the control compound with TNF $\alpha$.

Figure 3: 200 ns trajectory analysis of phytochemicals and control drug. A) RMSD, B) Rg, C) RMSF.

Figure 3B is a plot of radius of gyration for the trajectories over $200 \mathrm{~ns}$. It revealed similar structural compactness of all the phytochemicals and the control (average- $1.92 \mathrm{~nm}$ ). Interesting observations were made from the RMSF plot (Figure 3C). The average RMSF of the phytochemical complexes were like that of the control TNF complex (average: $0.14 \mathrm{~nm}$ ). Only the complex with Nimbolin A showed a slightly lower RMSF (average: $0.12 \mathrm{~nm}$ ). Analysis of the RMSF of the interface residues of chain A and chain B observed to interact with the phytochemicals and the control drug post docking has been presented in Table 3 and as bar chart in Figure 4.

Table 3: RMSF of C $\alpha$ of residues interacting with the phytochemicals and the control 


\begin{tabular}{|c|c|c|c|c|c|c|c|}
\hline 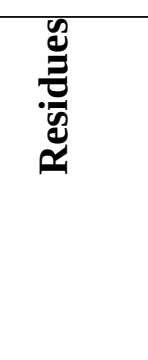 & 寻 & 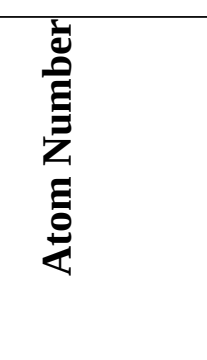 & 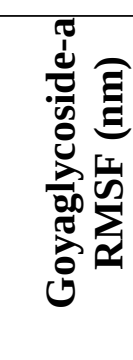 & 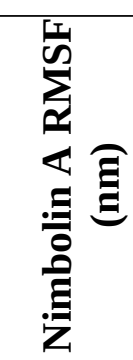 & 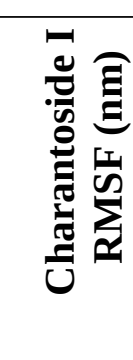 & 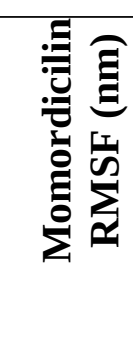 & 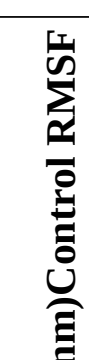 \\
\hline Leu 57 & $\mathrm{~A}$ & $647-665$ & 0.15 & 0.10 & 0.13 & 0.12 & 0.12 \\
\hline Ile58 & $A$ & $666-684$ & 0.10 & 0.12 & 0.11 & 0.10 & 0.13 \\
\hline Tyr 59 & $A$ & $685-703$ & 0.08 & 0.09 & 0.08 & 0.11 & 0.11 \\
\hline Lys98 & $\mathrm{A}$ & 1299-1320 & 0.14 & 0.11 & 0.11 & 0.11 & 0.11 \\
\hline Tyr119 & $A$ & 1463-1483 & 0.14 & 0.10 & 0.19 & 0.08 & 0.17 \\
\hline Tyr151 & $\mathrm{A}$ & 1955-1975 & 0.08 & 0.07 & 0.07 & 0.08 & 0.08 \\
\hline Gly153 & A & 1996-2002 & 0.06 & 0.05 & 0.05 & 0.06 & 0.06 \\
\hline Leu157 & A & $2056-2070$ & 0.18 & 0.18 & 0.15 & 0.15 & 0.14 \\
\hline Leu57 & $\mathrm{B}$ & $2799-2817$ & 0.13 & 0.11 & 0.13 & 0.09 & 0.12 \\
\hline Tyr59 & $\mathrm{B}$ & $2840-2957$ & 0.08 & 0.07 & 0.07 & 0.07 & 0.07 \\
\hline Lys98 & $\mathrm{B}$ & $3451-3472$ & 0.12 & 0.11 & 0.12 & 0.11 & 0.11 \\
\hline Pro117 & $\mathrm{B}$ & $3645-3658$ & 0.07 & 0.06 & 0.06 & 0.06 & 0.06 \\
\hline Tyr119 & B & $3678-3698$ & 0.05 & 0.05 & 0.05 & 0.05 & 0.06 \\
\hline Leu120 & B & $3699-3717$ & 0.07 & 0.08 & 0.07 & 0.08 & 0.08 \\
\hline Gly121 & B & $3718-3724$ & 0.06 & 0.06 & 0.06 & 0.05 & 0.06 \\
\hline Tyr151 & B & $4170-4188$ & 0.05 & 0.04 & 0.05 & 0.05 & 0.05 \\
\hline
\end{tabular}

Figure 4: RMSF of residues interacting with the phytochemicals and the control.

From Table 3 and Figure 4 it can be observed that binding of Goyaglycoside-a leads to reduction in fluctuation of residue Tyr 59 A compared to the other phytochemicals and the control. Nimbolin A results in reduction of fluctuation of most of the interface residues and reduction in fluctuation in Tyr119 A was significant with Momordicilin and Nimbolin A. Binding of Charantoside I do not show any significant reduction in fluctuation of any residues. It can be said here that as these residues are important for oligomerization, the binding of these phytochemicals that reduces its fluctuation, lower than the control inhibitor, may interfere with TNF $\alpha$ oligomerization as observed with the control ligand.

Hydrogen bond analysis over 200 ns trajectories revealed the residues with highest hydrogen bond occupancies were Tyr151 for the control inhibitor (24\% occupancy), Gly121 for Goyaglycoside-a (36\%) and Charantoside I (13\%), Tyr59 for Nimbolin A (24.4\%) and Momordicilin (24.4\%). For all the phytochemicals, Tyr59 and Tyr119 hydrogen bond occupancy was calculated to range from 8-23\%. Tyr119 and Gly121 were observed to be the residues redundantly involved in hydrogen bonds with the phytochemicals and the control 
inhibitor. Among the phytochemicals, Momordicilin was observed to possess higher hydrogen bond occupancies over the simulation time frame. Therefore, it can be expected that Momordicilin and TNF $\alpha$ complex will be more stable compared to the control and the other phytochemicals.

\subsection{PCA and DCCM analysis of the trajectories}

To extract the structural variations in detail upon the binding of the phytochemicals and the control drug, principal component analysis (PCA) on the C $\alpha$ atom was performed (Figure 5). The first five eigen vectors captured around $\sim 50 \%$ of the motions. The residue fluctuations were comparable to the control complex. Interestingly Nimbolin A showed lower fluctuations in comparison to the control and the other phytochemical complexes. This corroborates with the RMSF values observed for the residues in the interface of chain A and B (Figure 4). Furthermore, to understand the effect of phytochemicals binding on the internal dynamics of TNF $\alpha$, the dynamic cross-correlation matrix (DCCM) was calculated by using the coordinates of $\mathrm{C} \alpha$ atoms from the trajectories. DCCM analysis (Figure 6) showed that overall correlation increased, and anti- correlation decreased on binding of Nimbolin A and Momordicilin to TNF $\alpha$ in comparison to the control and the other two phytochemicals. Thus, it can be said that Nimbolin A and Momordicilin creates a more stable environment compared to the binding of the other phytochemicals and the control inhibitor.

Figure 5: PCA and residue fluctuation in PCA analysis of TNF $\alpha$ in complex with top scoring four phytochemicals. A) Control, B) Goyaglycoside-a, C) Charantoside I, D) Nimbolin A, E) Momordicilin

Figure 6: DCCM analysis of TNF $\alpha$ in complex with top scoring four phytochemicals. A) Control, B) Goyaglycoside-a, C) Charantoside I, D) Nimbolin A, E) Momordicilin.

\subsection{Relative binding free energy of phytochemicals and control inhibitor from $200 \mathrm{~ns}$ trajectories}

The relative binding free energy were calculated for each complex of phytochemicals and the control using MM-PBSA (Table 4). The standard errors were calculated by 500 steps of bootstrap analysis. It was observed that the relative binding free energies obtained for the 
complexes agreed with the RMSD, RMSF, PCA and DCCM calculations. Lowest relative binding energy was obtained for Momordicilin and TNF $\alpha$ complex (-190.5 kJ/Mol). Nimbolin A and the control inhibitor had comparable relative binding free energies, -188.03 and $-187.67 \mathrm{~kJ} / \mathrm{Mol}$ respectively.

Table 4: Relative binding energy calculated by MM/PBSA

\begin{tabular}{|c|c|c|c|c|c|}
\hline & $\begin{array}{l}\text { van der } \\
\text { Waal energy } \\
(\mathrm{kJ} / \mathrm{Mol})\end{array}$ & $\begin{array}{l}\text { Electrostatic } \\
\text { energy } \\
(\mathbf{k J} / \mathbf{M o l})\end{array}$ & $\begin{array}{l}\text { Polar } \\
\text { solvation } \\
\text { energy (kJ/ } \\
\text { Mol) }\end{array}$ & $\begin{array}{l}\text { SASA } \\
\text { energy } \\
(\mathbf{k J} / \mathbf{M o l})\end{array}$ & $\begin{array}{l}\text { Binding } \\
\text { energy } \\
(\mathrm{kJ} / \mathrm{Mol})\end{array}$ \\
\hline Goyaglycoside-a & -235.81 & -6.55 & 86.535 & -22.576 & -178.402 \\
\hline *Std Error & $+/-16.755$ & $+/-\quad 3.348$ & $+/-12.245$ & $+/-\quad 1.432$ & $+/-15.092$ \\
\hline Charantoside I & -219.158 & -7.102 & 96.189 & -22.004 & -152.074 \\
\hline Std Error & $+/-15.823$ & $+/-\quad 3.644$ & $+/-15.026$ & $\begin{array}{ll}+/- & 1.426 \\
\end{array}$ & $+/-\quad 17.398$ \\
\hline Nimbolin A & -237.253 & -2.548 & 72.794 & -21.024 & -188.032 \\
\hline Std Error & $+/-\quad 12.418$ & $\begin{array}{ll}+/- & 1.391 \\
\end{array}$ & $+/-\quad 7.032$ & $+/-\quad 1.016$ & $+/-\quad 11.950$ \\
\hline Momordicilin & -239.086 & -1.491 & 71.683 & -21.597 & -190.492 \\
\hline Std Error & $+/-\quad 13.092$ & $+/-\quad 1.219$ & $+/-\quad 6.636$ & $\begin{array}{ll}+/- & 1.028 \\
\end{array}$ & $+/-12.481$ \\
\hline Control & -224.41 & -0.902 & 58.664 & -21.023 & -187.672 \\
\hline Std Error & $+/-\quad 18.589$ & $+/-\quad 1.404$ & $+/-8.950$ & $\begin{array}{ll}+/- & 1.684\end{array}$ & $+/-17.961$ \\
\hline
\end{tabular}

*The standard error (Std Error) was calculated after 500 step bootstrap analysis.

Residue decomposition for their contribution towards the relative binding energy showed that Leu57, Tyr59 and Tyr119 were the lowest relative free energy contributors ( 7$10 \mathrm{~kJ} / \mathrm{Mol}$ ). These residues were also observed to have lower fluctuations as analysed by the RMSF, PCA and DCCM calculations.

\subsection{Free energy landscape and network centrality analysis}

Free energy landscapes (FEL) were constructed using the projections of first (PC1) and second (PC2) eigenvectors using g_sham tool of GROMACS. The contour maps of Momordicilin and Nimbolin A complexes showed similar energy clusters as the control. The global minimum energy conformations were obtained from FEL bins at 200ps for control, 150 ns for Momordicilin and 52 ns for Nimbolin A (Figure 7). FEL revealed $\Delta G$ value 0 to 11, 12.7 and $11.4 \mathrm{~kJ} / \mathrm{mol}$ for control, Momordicilin and Nimbolin A complexes respectively with TNF $\alpha$. The conformations found in the blue area are more stable than the red area. Different global minima were observed during the 200 ns MD simulations for the control and the phytochemical complexes with TNF $\alpha$. Several minima were observed corresponding to the metastable conformational states separated with small energy barriers. 
Figure 7: Free energy landscape contour maps and network centrality analysis of the structure with minimum energy obtained from FEL. Red: Higher Gibbs free energy, Blue: Lower Gibbs free energy.

The global energy minimum conformation obtained from FEL bin were subjected to network centrality analysis. Weighted network analysis with atom pair contact and upper threshold of $5 \AA$ were constructed and the centrality measures of betweenness centrality, degree centrality and interaction strength were compared among the structures (Figure 7). It was observed that the degree, betweenness centrality and interaction strength of residues near the ligand binding site were like the control, being slightly higher for the TNF $\alpha$ complex with Momordicilin, which was also calculated to have the lowest relative binding free energy. This indicates that the phytochemicals can become potent inhibitors of TNF $\alpha$. It was also observed that degree, betweenness centrality and residue interaction strength increased for residues Lys98, Tyr119 and Tyr151 in case of Momordicilin complex.

\section{Discussions}

The aim of this project was to identify phytochemicals as inhibitors of TNF $\alpha$. TNF $\alpha$ was chosen as a drug target as it is the therapeutic target for various inflammatory and autoimmune related diseases [1]. More specifically phytochemicals that can lead to deoligomerisation of TNF $\alpha$ similar to the inhibitors reported by He et al. [18] from Sunesis Pharmaceuticals, USA [9,18]. A total of 6000 compounds were screened for TNF $\alpha$. It was interesting to observe that phytochemicals from Momordica charantia, Azadirachta indica, Withania somnifera, Mangifera indica, Euphorbia hirta and Nigella sativa were screened to have potential binding affinity- comparable to the control for TNF $\alpha$ dimer binding site. In traditional medicine Momordica charantia has been used as antiviral, anti-malarial, and antibacterial agent. It is also reported to be anti-diabetic [48].

Further analysis revealed that the top 10 scoring compounds with docking score $<-10$ kJ/Mol were from Momordica charantia, Azadirachta indica, Strychnos nux-vomica, Bauhinia purpurea, Swertia chirayita, Solanum torvum and Mangifera indica. The results corroborate with the report where Momordica charantia was reported to have antiinflammatory effect in sepsis mice and reduced the levels of cytokines IL-1, IL-6, and TNF- $\alpha$ 
[49]. Interestingly Momordica charantia fruit extract was reported to have protective effect on TNF $\alpha$-induced NF-кB activation and cardiomyocyte apoptosis [50]. After identifying the top 10 docked complexes having docking score to be more negative than the control was subjected to toxicity analysis. The control inhibitor (ligand PDB ID: 307) was extracted from the crystal structure of TNF $\alpha$ (2az5). Four phytochemicals having low toxicity profile, docking score $<-10 \mathrm{~kJ} / \mathrm{Mol}$ and the control inhibitor were subjected to all atom molecular dynamics simulation of 200 ns to establish the binding stability of the phytochemicals in comparison to the control. The selected four phytochemicals were Goyaglycoside-a, Charantoside I, Nimbolin A and Momordicilin. The stability analysis by RMSD, RMSF and Rg revealed that the phytochemicals formed stable interaction with the binding site of TNF $\alpha$. PCA and DCCM analysis showed that a more stable environment was created by binding of Nimbolin A and Momordicilin in comparison to the control and the other two phytochemicals. This was evident by decreased fluctuation of the binding site residues and increased correlation in the residues of TNF $\alpha$. Interestingly calculation of relative binding free energy over the 200 ns simulation trajectories revealed that Nimbolin A and Momordicilin were with more negative relative binding free energy compared to other two phytochemicals and like the control. Therefore, it can be said that these phytochemicals can be potential inhibitors for causing TNF $\alpha$ de-oligomerization. Analysis of residue interaction with these phytochemicals showed that Tyr59 and Tyr119 hydrogen bond occupancy was calculated to be 8-23\%. Tyr119, Gly121 were observed to be the residues involved in hydrogen bonds with the phytochemicals and the control inhibitor. Residue decomposition analysis for contributing towards the relative free binding energy showed that Leu57, Tyr59 and Tyr119 were the lowest relative free energy contributors $(\sim 7-10 \mathrm{~kJ} / \mathrm{Mol})$.

Interestingly network analysis performed on the global energy minimum conformation also showed that the degree, betweenness centrality and interaction strength were similar for the control, Momordicilin and Nimbolin A complexes. There was slight increase in centrality measures in Lys98, Tyr119 and Tyr151 in case of Momordicilin complex. This indicates TNF $\alpha$ formed stable complexes with these phytochemicals. This also corroborates with earlier report where small molecule was reported to interact with Tyr119 resulting in disruption of TNF trimer [18]. These residues were also reported to interact with capsazepine derivatives [51]. Therefore, these phytochemicals can be potential inhibitors of TNF preventing its oligomerization and interaction with TNF-R1.

\section{Conclusions}


In conclusion the master regulator for inflammatory pathways in human, TNF $\alpha$, was used as a drug target to screen phytochemical inhibitors in machino. The aim was to identify potent phytochemicals that can bind to TNF $\alpha$ dimer at a site that causes inhibition of oligomerization and prevention of its binding to TNF-R1 receptor. Two potential triterpenoid phytochemicals from Momordica charantia and Azadirachta indica; namely Momordicilin and Nimbolin A respectively were identified from 6000 phytochemicals to be potential inhibitors. The stability of the inhibitors was established by performing 200 ns molecular dynamics simulations. The relative binding free energies were calculated to be $-190.5 \mathrm{~kJ} / \mathrm{Mol}$ for Momordicilin and $-188.03 \mathrm{~kJ} / \mathrm{Mol}$ for Nimbolin A. Conclusively these phytochemicals can be used for further in vitro analysis to confirm their inhibitory efficacy against TNF $\alpha$. Conclusively, this work paves the way for a class of phytochemicals capable of modulating TNF $\alpha$ function probably by trimer destabilization.

\section{ACKNOWLEDGEMENT}

We would like to acknowledge Noor Enzymes Private Limited for providing us with the human resources needed to successfully complete this work.

\section{CONFLICT OF INTEREST}

No potential conflict of interest was reported by the authors.

\section{AUTHOR CONTRIBUTIONS STATEMENT}

Pratap Kumar Parida: Methodology, Software, Formal Analysis. Dipak Paul: Data curation, Writing- Original draft preparation. Debamitra Chakravorty: Conceptualization, Visualization, Investigation, Supervision, Validation, Writing- Reviewing and Editing. All data were generated in-house, and no paper mill was used. All authors agree to be accountable for all aspects of work ensuring integrity and accuracy.

\section{SUPPLEMENTAL ONLINE MATERIAL}

Supplemental data for this article is available online.

\section{ORCID}


Pratap Kumar Parida: 0000-0002-8193-335X

Dipak Paul: 0000-0002-5664-4601

Debamitra Chakravorty: 0000-0002-1520-0780

\section{References}

[1] M.S. Saddala, H. Huang, Identification of novel inhibitors for TNF $\alpha$, TNFR1 and TNF $\alpha-$ TNFR1 complex using pharmacophore-based approaches, J. Transl. Med. 17 (2019) 215. https://doi.org/10.1186/s12967-019-1965-5

[2] H.T. Idriss, J.H. Naismith, TNF $\alpha$ and the TNF receptor superfamily: structure-function relationship(s), Microsc. Res. Tech. 50 (3) (2000) 184-195. https://doi.org/10.1002/10970029(20000801)50:3<184::AID-JEMT2>3.0.CO;2-H

[3] C. Brightling, M. Berry, Y. Amrani, Targeting TNF- $\alpha$ : a novel therapeutic approach for asthma, J. Allergy Clin. Immunol. $121 \quad$ (1) (2008) 5-10. https://doi.org/10.1016/j.jaci.2007.10.028

[4] H. Loetscher, Y.C. Pan, H.W. Lahm, R. Gentz, M. Brockhaus, H. Tabuchi, W. Lesslauer, Molecular cloning and expression of the human $55 \mathrm{kd}$ tumor necrosis factor receptor, Cell 61 (2) (1990) 351-359. https://doi.org/10.1016/0092-8674(90)90815-V

[5] L. Cabal-Hierro, P.S. Lazo, Signal transduction by tumor necrosis factor receptors, Cell Signal 24 (6) (2012) 1297-1305. https://doi.org/10.1016/j.cellsig.2012.02.006

[6] D. Tracey, L. Klareskog, E.H. Sasso, J.G. Salfeld, P.P. Tak, Tumor necrosis factor antagonist mechanisms of action: a comprehensive review, Pharmacol. Ther. 117 (2) (2008) 244-279. https://doi.org/10.1016/j.pharmthera.2007.10.001

[7] A. Caminero, M. Comabella, X. Montalban, Tumor necrosis factor alpha (TNF- $\alpha$ ), antiTNF- $\alpha$ and demyelination revisited: an ongoing story, J. Neuroimmunol. 234 (1-2) (2011) 16. https://doi.org/10.1016/j.jneuroim.2011.03.004

[8] J. O'Connell, J. Porter, B. Kroeplien, T. Norman, S. Rapecki, R. Davis, D. McMillan, T. Arakaki, A. Burgin, D. Fox III, T. Ceska, F. Lecomte, A. Maloney, A. Vugler, B. Carrington, B.P. Cossins, T. Bourne, A. Lawson, Small molecules that inhibit TNF signalling by stabilising an asymmetric form of the trimer, Nat. Commun. 10 (2019) 5795. https://doi.org/10.1038/s41467-019-13616-1

[9] J.M. Davis, J. Colangelo, Small-molecule inhibitors of the interaction between TNF and TNFR, Future Med. Chem. 5 (1) (2013) 69-79. https://doi.org/10.4155/fmc.12.192

[10] E. Douni, G. Kollias, A critical role of the p75 tumor necrosis factor receptor (p75TNF$\mathrm{R}$ ) in organ inflammation independent of TNF, lymphotoxin alpha, or the p55TNF-R, J. Exp. Med. 188 (7) (1998) 1343-1352. https://doi.org/10.1084/jem.188.7.1343 
[11] D. Kontoyiannis, G. Boulougouris, M. Manoloukos, M. Armaka, M. Apostolaki, T. Pizarro, A. Kotlyarov, I. Forster, R. Flavell, M. Gaestel, P. Tsichlis, F. Cominelli, G. Kollias, Genetic dissection of the cellular pathways and signaling mechanisms in modeled tumor necrosis factor-induced Crohn's-like inflammatory bowel disease, J. Exp. Med. 196 (12) (2002) 1563-1574. https://doi.org/10.1084/jem.20020281

[12] K.W. Choi, H.J. Park, D.H. Jung, T.W. Kim, Y.M. Park, B.O. Kim, E.H. Sohn, E.Y. Moon, S.H. Um, D.K. Rhee, S. Pyo, Inhibition of TNF- $\alpha$-induced adhesion molecule expression by diosgenin in mouse vascular smooth muscle cells via downregulation of the MAPK, Akt and NF-kB signaling pathways, Vascul. Pharmacol. 53 (5-6) (2010) 273-280. https://doi.org/10.1016/j.vph.2010.09.007

[13] G. Melagraki, E. Ntougkos, D. Papadopoulou, V. Rinotas, G. Leonis, E. Douni, A. Afantitis, G. Kollias, In silico discovery of plant-origin natural product inhibitors of tumor necrosis factor (TNF) and receptor activator of NF-kB ligand (RANKL), Front. Pharmacol. 9 (2018) 800. https://doi.org/10.3389/fphar.2018.00800

[14] L. Grazioli, R. Alzani, M. Ciomei, M. Mariani, A. Restivo, E. Cozzi, F. Marcucci, Inhibitory effect of suramin on receptor binding and cytotoxic activity of tumor necrosis factor $\alpha$, Int. J. Immunopharmacol. 14 (4) (1992) 637-642. https://doi.org/10.1016/0192$\underline{0561(92) 90125-5}$

[15] R. Alzani, A. Corti, L. Grazioli, E. Cozzi, P. Ghezzi, F. Marcucci, Suramin induces deoligomerization of human tumor necrosis factor alpha, J. Biol. Chem. 268 (17) (1993) 12526-12529. https://doi.org/10.1016/S0021-9258(18)31420-0

[16] F. Mancini, C.M. Toro, M. Mabilia, M. Giannangeli, M. Pinza, C Milanese, Inhibition of tumor necrosis factor- $\alpha$ (TNF- $\alpha$ )/TNF- $\alpha$ receptor binding by structural analogues of suramin. Biochem. Pharmacol. 58 (5) (1999) 851-859. https://doi.org/10.1016/S0006-2952(99)00150$\underline{1}$

[17] Y. Mukai, T. Nakamura, M. Yoshikawa, Y. Yoshioka, S. Tsunoda, S. Nakagawa, Y. Yamagata, Y. Tsutsumi, Solution of the structure of the TNF-TNFR2 complex, Sci. Signal. 3 (148) (2010) ra83. https://doi.org/10.1126/scisignal.2000954

[18] M.M He, A.S. Smith, J.D. Oslob, W.M. Flanagan, A.C. Braisted, A. Whitty, M.T. Cancilla, J. Wang, A.A. Lugovskoy, J.C. Yoburn, A.D. Fung, G. Farrington, J.K. Eldredge, E.S. Day, L.A. Cruz, T.G. Cachero, S.K. Miller, J.E. Friedman, I.C. Choong, B.C. Cunningham, Small-molecule inhibition of TNF- $\alpha$, Science 310 (5750) (2005) 1022-1025. https://doi.org/10.1126/science.1116304

[19] P. Alexiou, A. Papakyriakou, E. Ntougkos, C.P. Papaneophytou, F. Liepouri, A. Mettou, I. Katsoulis, A. Maranti, K. Tsiliouka, A. Strongilos, S. Chaitidou, Rationally designed less toxic SPD-304 analogs and preliminary evaluation of their TNF inhibitory effects, Arch. Pharm. 347 (11) (2014) 798-805. https://doi.org/10.1002/ardp.201400198 
[20] F. Buller, Y. Zhang, J. Scheuermann, J. Schäfer, P. Bühlmann, D. Neri, Discovery of TNF inhibitors from a DNA-encoded chemical library based on diels-alder cycloaddition, Chem. Biol. 16 (10) (2009) 1075-1086. https://doi.org/10.1016/j.chembiol.2009.09.011

[21] B.A. Shah, R. Chib, P. Gupta, V.K. Sethi, S. Koul, S.S. Andotra, A. Nargotra, S. Sharma, A. Pandey, S. Bani, B. Purnima, S.C. Taneja, Saponins as novel TNF- $\alpha$ inhibitors: isolation of saponins and a nor-pseudoguaianolide from Parthenium hysterophorus, Org. Biomol. Chem. 7 (16) (2009) 3230-3235. https://doi.org/10.1039/b902041a

[22] M.D.C. Recio, R.M. Giner, S. Máñez, J.L. Ríos, Structural requirements for the antiinflammatory activity of natural triterpenoids, Planta Medica 61 (2) (1995) 182-185. https://doi.org/10.1055/s-2006-958045

[23] D.S. Chan, H.M. Lee, F. Yang, C.M. Che, C.C. Wong, R. Abagyan, C.H. Leung, D.L. Ma, Structure-based discovery of natural-product-like TNF- $\alpha$ inhibitors, Angew. Chem. Int. Ed. Engl. 49 (16) (2010) 2860-2864. https://doi.org/10.1002/anie.200907360

[24] S.T. Wua, J.C. Suna, K.J. Leeb, Y.M. Sunc, Docking prediction for tumor necrosis factor- $\alpha$ and five herbal inhibitors, Int. J. Eng. Sci. Technol. 2 (2010) 4263-4277.

[25] M. Reutlinger, T. Rodrigues, P. Schneider, G. Schneider, Multi-objective molecular de novo design by adaptive fragment prioritization, Angew. Chem. Int. Ed. Engl. 53 (16) (2014) 4244-4248. https://doi.org/10.1002/anie.201310864

[26] T. Rodrigues, D. Reker, P. Schneider, G. Schneider, Counting on natural products for drug design, Nat. Chem. 8 (6) (2016) 531. https://doi.org/10.1038/nchem.2479

[27] S. Basith, M. Cui, S.J. Macalino, J. Park, N.A. Clavio, S. Kang, S. Choi, Exploring G protein-coupled receptors (GPCRs) ligand space via cheminformatics approaches: impact on $\begin{array}{llllll}\text { rational drug design, } & \text { Front. } & \text { Pharmacol. } & 9 & \text { (2018) } & 128 .\end{array}$ https://doi.org/10.3389/fphar.2018.00128

[28] M.N. Lima, C.C. Melo-Filho, G.C. Cassiano, B.J. Neves, V.M. Alves, RC Braga, P.V. Cravo, E.N. Muratov, J. Calit, D.Y. Bargieri, F. Costa, QSAR-driven design and discovery of novel compounds with antiplasmodial and transmission blocking activities, Front. Pharmacol. 9 (2018) 146. https://doi.org/10.3389/fphar.2018.00146

[29] S. Zheng, M. Jiang, C. Zhao, R. Zhu, Z. Hu, Y. Xu, F. Lin, e-Bitter: bitterant prediction by the consensus voting from the machine-learning methods, Front. Chem. 6 (2018) 82. https://doi.org/10.3389/fchem.2018.00082

[30] R.A. Laskowski, PDBsum new things, Nucleic Acids Res. 37 (2009) D355-D359. https://doi.org/10.1093/nar/gkn860

[31] Dassault Systèmes BIOVIA, BIOVIA Discovery Studio Visualiser, v20.1..0.19295, San Diego, CA, USA, 2020. 
[32] A. Daina, O. Michielin, V. Zoete, iLOGP: a simple, robust, and efficient description of n-octanol/water partition coefficient for drug design using the GB/SA approach, J. Chem. Inf. Model. 54 (12) (2014) 3284-3301. https://doi.org/10.1021/ci500467k

[33] A. Daina, V. Zoete, A BOILED-Egg to predict gastrointestinal absorption and brain penetration of small molecules, ChemMedChem 11 (11) (2016) 1117-1121. https://doi.org/10.1002/cmdc.201600182

[34] A. Daina, O. Michielin, V. Zoete, SwissADME: a free web tool to evaluate pharmacokinetics, drug-likeness and medicinal chemistry friendliness of small molecules, Sci. Rep. 7 (2017) 42717. https://doi.org/10.1038/srep42717

[35] P. Schyman, R. Liu, V. Desai, A. Wallqvist, vNN web server for ADMET predictions, Front. Pharmacol. 8 (2017) 889. https://doi.org/10.3389/fphar.2017.00889

[36] C.A. Lipinski, F. Lombardo, B.W. Dominy, P.J. Feeney, Experimental and computational approaches to estimate solubility and permeability in drug discovery and development settings, Adv. Drug Deliv. Rev. $23 \quad$ (1-3) (1997) 3-25. https://doi.org/10.1016/S0169-409X(96)00423-1

[37] J.B. Baell, G.A. Holloway, New substructure filters for removal of pan assay interference compounds (PAINS) from screening libraries and for their exclusion in bioassays, J. Med. Chem. 53 (7) (2010) 2719-2740. https://doi.org/10.1021/jm901137j

[38] R. Brenk, A. Schipani, D. James, A. Krasowski, I.H. Gilbert, J. Frearson, P.G. Wyatt, Lessons learnt from assembling screening libraries for drug discovery for neglected diseases, ChemMedChem 3 (3) (2008) 435-444. https://doi.org/10.1002/cmdc.200700139

[39] S.J. Teague, A.M. Davis, P.D. Leeson, T. Oprea, The design of leadlike combinatorial libraries, Angew. Chem. Int. Ed. Engl. $38 \quad$ (24) (1999) 3743-3748. https://doi.org/10.1002/(SICI)1521-3773(19991216)38:24<3743::AID-ANIE3743>3.0.CO;2$\underline{\mathrm{U}}$

[40] Lindahl, Abraham, Hess, van der Spoel, GROMACS 2020.2 Source code, Zenodo, 2020. http://doi.org/10.5281/zenodo.3773801

[41] A.W. Da Silva, W.F. Vranken, ACPYPE-Antechamber python parser interface, BMC Res. Notes 5 (2012) 367. https://doi.org/10.1186/1756-0500-5-367

[42] T. Darden, D. York, L. Pedersen, Particle mesh Ewald: An N • log (N) method for Ewald sums in large systems, J. Chem. Phys. 98 (12) (1993) 10089-10092. https://doi.org/10.1063/1.464397

[43] B.J. Grant, A.P. Rodrigues, K.M. ElSawy, J.A. McCammon, L.S. Caves, Bio3d: an R package for the comparative analysis of protein structures, Bioinformatics 22 (21) (2006) 2695-2696. https://doi.org/10.1093/bioinformatics/btl461 
[44] K. Al-Khafaji, T. Taskin Tok, Amygdalin as multi-target anticancer drug against targets of cell division cycle: double docking and molecular dynamics simulation, J. Biomol. Struct. Dyn. (2020). https://doi.org/10.1080/07391102.2020.1742792

[45] N.A. Baker, D. Sept, S. Joseph, M.J. Holst, J.A. McCammon, Electrostatics of nanosystems: application to microtubules and the ribosome, Proc. Natl. Acad. Sci. 98 (18) (2001) 10037-10041. https://doi.org/10.1073/pnas.181342398

[46] R. Kumari, R. Kumar, Open Source Drug Discovery Consortium, A. Lynn, g_mmpbsa A GROMACS tool for high-throughput MM-PBSA calculations, J. Chem. Inform. Model. 54 (7) (2014) 1951-1962. https://doi.org/10.1021/ci500020m

[47] M.J. Eck, S.R. Sprang, The structure of tumor necrosis factor-alpha at 2.6 A resolution. Implications for receptor binding, J. Biol. Chem. 264 (29) (1989) 17595-17605. https://doi.org/10.2210/pdb1tnf/pdb

[48] J.K. Grover, S.P. Yadav, Pharmacological actions and potential uses of Momordica charantia: a review, J. Ethnopharmacol. $93 \quad$ (1) (2004) 123-132. https://doi.org/10.1016/j.jep.2004.03.035

[49] C.Y. Chao, P.J. Sung, W.H. Wang, Y.H. Kuo, Anti-inflammatory effect of Momordica charantia in sepsis mice, Molecules 19 (8) (2014) 12777-12788. https://doi.org/10.3390/molecules190812777

[50] H. Hu, Y. Liu, B. Kong, Y. Fan, Z. Wang, J. Peng, X. Xiong, Y. Mei, W. Liu, H. Huang, Protective effect of Momordica charantia fruit extract on TNF $\alpha$-induced NF-кB activation and cardiomyocyte apoptosis, Int. J. Clin. Exp. Med. 9 (3) (2016) 5951-5959.

[51] A. Shukla, P. Sharma, O. Prakash, M. Singh, K. Kalani, F. Khan, D.U. Bawankule, S. Luqman, S.K. Srivastava, QSAR and docking studies on capsazepine derivatives for immunomodulatory and anti-inflammatory activity, PLoS One 9 (7) (2014) e100797. https://doi.org/10.1371/journal.pone.0100797

\section{List of Tables}

Table 1: Top 10 phytochemicals docked to TNF $\alpha$

Table 2: Molecular interactions obtained after docking of phytochemicals and control drug with the homodimer of TNF $\alpha$

Table 3: RMSF of C $\alpha$ of residues interacting with the phytochemicals and the control

Table 4: Relative binding energy calculated by MM/PBSA

\section{List of Figures}


Figure 1: TNF $\alpha$ in inflammation related diseases. The signalling pathway was adapted from KEGG. The diseases associated have been illustrated in blue circles. The biologics available for TNF are illustrated in orange circle. The cartoon representation of the structure of TNF $\alpha$ in complex with a small molecule inhibitor (represented in cyan ball and stick) was obtained using PyMol molecular graphics tool. The bar graph depicts the \% of compounds from the medicinal plants that docked to TNF $\alpha$ with docking score lesser than the bound control ligand obtained from RCSB PDB (PDB ID: 2az5).

Figure 2: Docked complexes and residue interaction 2D image of phytochemicals and control drug with TNF $\alpha$ having lead likeness 3. A) Control, B) Goyaglycoside-a, C) Charantoside I, D) Nimbolin A, E) Momordicilin.

Figure 3: 200 ns trajectory analysis of phytochemicals and control drug. A) RMSD, B) Rg, C) RMSF.

Figure 4: RMSF of residues interacting with the phytochemicals and the control.

Figure 5: PCA and residue fluctuation in PCA analysis of TNF $\alpha$ in complex with top scoring four phytochemicals. A) Control, B) Goyaglycoside-a, C) Charantoside I, D) Nimbolin A, E) Momordicilin

Figure 6: DCCM analysis of TNF $\alpha$ in complex with top scoring four phytochemicals.A) Control, B) Goyaglycoside-a, C) Charantoside I, D) Nimbolin A, E) Momordicilin 